\title{
Performance Assessment of Industrial Drives due to Power Quality Issues and its Potential Impacts on Electrical Drives
}

\author{
Vishnu Murthy $\mathrm{K}^{1}$, Ashok Kumar L ${ }^{2}$ \\ $\left\{\right.$ drkvm25@gmail.com ${ }^{1}$, lak.eee@psgtech.ac.in $\left.{ }^{2}\right\}$ \\ ${ }^{1}$ Research Scholar, ${ }^{2}$ Professor, Department of Electrical and Electronics Engineering, PSG College of \\ Technology, Coimbatore, Tamilnadu
}

\begin{abstract}
Power quality difficulties have been predicted since electronically powered drives have been the main component of any manufacturing process. Evaluating the same's informative quality has proven to be a big challenge. Because of non-linearity on the load side and an over-reliance on semiconductor technology, a good power quality could be crucial. The capabilities of the drives section employed in the business are also attracting attention in the current circumstances. As a result of the concerns mentioned above, several literary scientific studies show disturbances in the load side and the efficiency of electric drives. Weakness resulting from power quality is simply too significant, making it difficult for even the most sophisticated engineering to function effectively. A deep insight examination of grid side characteristics was performed in conjunction with investigating the inverter drive section responsiveness to grid variation to confirm the grid side issues for the artificial intelligence technique. This study work emphasized and proposed an answer to the possible artificial wise approach for administering fault deterrence prediction algorithm after clear proof from analysis and accounting grid side quality concerns relevant to electronic powered drives. The result is an accurate forecast of abnormal grid side behavior to offer safe and trustworthy grid operation, reducing the likelihood of shutdown or failure to a greater extent. Improvements in forecast accuracy based on previous data have been viable feedback to business equipment manufacturers, in addition to the aforementioned technique.
\end{abstract}

Keywords: Power Quality Issues, Inverter Drives Failure, Predictive Analysis, Inverter Drive Fault Prediction, Machine Learning Prediction Analysis..

\section{Introduction}

Artificial intelligence is currently a pervasive technology across all industries. It encompasses a broad range of disciplines, including computer science, computer engineering, economics, information science, software engineering, robotics, electrical engineering, and medical technology. It was not until recently, however, that computer scientists, roboticists, and software engineers were able to integrate this technology into manufacturing. The fastest growing topic is artificial intelligent systems, which are being used in a number of fields ranging from computer science to health to combat. Artificial intelligence is a generic term that refers to computers and other machines' ability to learn, adapt, and even multiply in order to accomplish various activities far more efficiently than normal humans. Inverter Drives, 
which employ similar concepts, are currently employed to regulate enormous industrial requirements.

While comprehending how this type of artificial intelligence works is tough, seeing how it works is not. Consider the Fr-E 800 series VFDs. These devices generate random, highfrequency vibrations that move industrial equipment components. In addition to generating sound and light, these types of vibrations are employed to regulate factory operations and generate enhanced signal quality. There are nearly endless applications for this technology, but they all entail the use of high-frequency energy to power machinery. To understand such technologies, it is necessary to first understand the VFDS, or Variable Frequency Drive. The vibrations produced by the motor are transformed to digital signals, which can be analysed by a variety of software applications. Typical applications include managing home appliances and automobiles. Numerous manufacturers of VFDS are now developing computer software that will enable consumers to control their own household appliances with a few buttons clicks. This cutting-edge technology has the potential to fundamentally alter the way we work. For instance, in the not-too-distant future, each domestic appliance will be equipped with its own computer and battery. This type of setup will require the VFDS.

Even microwaves and radios can be changed with the help of VFDs. However, they can not only be used to replace single appliances, but can also be used to drive more sophisticated machines. Using this software, machines are able to communicate, drive, and do a variety of other tasks It's possible that artificial intelligence computers will one day be able to execute tasks that would ordinarily be performed by a human using a simple computer. Using a electrical circuits to perform things that were previously only feasible with supercomputers is amazing.

\section{Technical Survey of Current Industrial Drives - A Broad Overview}

While conducting research on several manufacturing issues, the simplest component to begin with is assuming that normal voltage and associated parametric concerns are truly specified and continue along with other system bottlenecks. In this regard, the most significant elements may be a lengthy hour of argument that convincing analyses are genuinely necessary since the input side voltage and other grid characteristics are not up to IEEE and IEC standards [1][2]. Austerity in a faulty signal state from the grid to the drive platform results in a temporary or permanent failure, or even deteriorated scenarios inside the drive system. The length and magnitude of the specific parameter anomaly from the input supply side are also taken into account [3]. Sagging situations, in particular, may be caused by the energising of an energy transformer, a large capacity load, an occurrence of lighting, a short circuit, or a combination of the foregoing [4]. Thermal behaviour aspects also have an effect on the drive system's functioning, which has an effect on the insulation of ac systems and hence on their own system's reliability. As a result, a VFD should be selected in line with the specification, as it is critical in reducing the above-mentioned concerns on the drive and also on the ac machine [5]. This research [6] cites the GOST pattern for detecting various energy quality concerns such as swell, sag, and disruptions by utilising the root mean square of that particular signal during the specified time period. To possess the aforementioned ways, the adverse event identified is also minimised, hence minimising the financial impact [6].

The AC induction with capacitor banks is in danger when the defined voltage level fluctuations fall below par value between $10 \%$ and $90 \%$ even during normal circumstances 
that last under a minute for roughly half the signal value. Despite the device's capacitor bank, it couldn't handle the transients caused by poor power quality. Because capacitor banks are designed to compensate for reactive power losses, they fail to perform as intended, resulting in higher reactive power flaws [7]. It's to look at the strain as a result of the exact same, thus manufacturers should look at this problem through the planning process [8]. Especially in three phase situations, it's difficult to keep all phase voltages exactly the same. According to NEMA standards, when phase voltage fluctuates, the motor's performance degrades [9]. Recovering procedures are employed to defray drooping situations, however reward may only be around $50 \%[10]$.

Again, [11]the sag quality finds function that's vital in analysing the diverse energy losses as a result of the same. Root mean square disturbances are major types of loose situations that influence industrial drives, and methods to repair these concerns were discussed. The study clarifies this by limiting voltage variation at the primary end or by restricting increase or DVR technology or restraining current rise of the AC electrical machine due to torque reduction. The increasing use of power semiconductor converters has had a visible influence on grid networks, introducing harmonics into the networks. Despite these efforts to reduce the said issues, a primary effect in what seems like a earlier case on power grid as per IEC [12]. Due to these actions within the device, the drives section heats up more, resulting in spark at the electrical machine. Due to the sustained increase in heating impact, injected harmonics will undoubtedly test the engine performance. The main reasons for cutting this material are current and voltage distortion, common mode voltage, and manufacturing design requirements. In this sense, thermal and common mode voltages are constantly monitored to maintain drive safety [13].

\section{Research Analysis on a Defined Fault Incident in Electric Drive Mechanisms.}

According to the findings of the literature review, the studies were documented within the drive equipment, which may have occurred as a result of abnormal load conditions or as a result of anomalous behaviour within the equipment. In addition, the defect caused by the grid network that has an impact on the drive performance parameters has received a lot of attention in the literature. However, it is difficult to locate because of an unattended defect in the grid, and the performance of a critical drive system continues to deteriorate rapidly. In this regard, an understanding of the fault event is critical, and the following paragraph of this research article discusses the analysis of electrical grid parameter behaviour using data gathered from the industrial sector. The analysis takes into account grid factors such as voltage, current, frequency, harmonics (both voltage and current parts), carbon emission, and instantaneous power.

3.1 Behaviour of Current Variables During a Voltage Flags Occurrence:

In the context of the fault occurrence moment or flag occurrence, voltage sag/swell plays a crucial role in current consumed by the electrical drives. The test device records pre- and post-flag occurrence vs. present behaviour, as illustrated in Figure 1. After flag event, the Yaxis shows the current value (Maximum, Average, and Minimum) for $1 / 2$ cycle. In the drive system, the current values majority jumps up whenever a flag event (F, O or P, O) occurs, which is abnormal behaviour in the grid network. It is clear from figure 1 that under scenarios of "O", that is, when nominal values are dominant, the current values recorded are anywhere 
over zero. Current either shoots to maximum value which is commonly reached in the system, or zero and average values are also recorded when F,O,P,O dominates the network. There are also times when the current is at zero or even the average of the usual operation while the flag ' $\mathrm{O}$ ' is on. When the nominal voltage is restored in the grid system after a flag event (i.e., post $\mathrm{F}, \mathrm{O}$ or $\mathrm{P}, \mathrm{O}$ ), the bulk of the current values stay close to the values recorded during the post event.

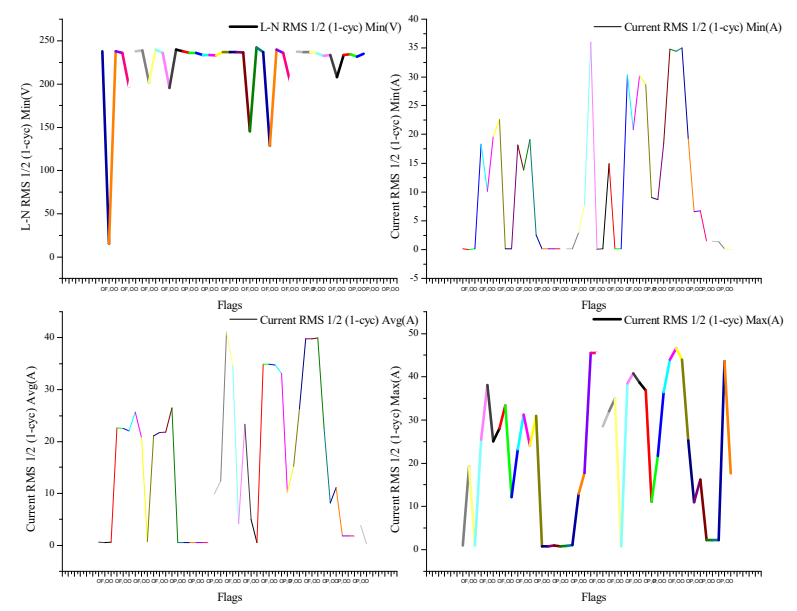

Figure 1. Flag Event vs Load Current Behaviour

3.2 Flag Occurrence in Relation to Three-Phase Voltage Variations:

Figure 2 shows the three-phase Line to Line and Line to Neutral network voltage analysis when the flag occurs in distinct phases. As shown in the picture, the grid power system maintains a nominal line voltage of $415 / 230 \mathrm{~V}$, both before and after the flag event has occurred. When the flagged moment $(\mathrm{F}, \mathrm{O})$ occurs, however, the voltage drops by around half of the time, while the other half of the time, the voltage stays near to the normal level. In the test phase, the labelling value for voltage minimum is recorded. This clearly shows that when the grid voltage falls below the par value, it has an influence on the drive system. The picture also shows that, when a phase is interrupted, it is not necessary for all voltage values across lines to be zero.

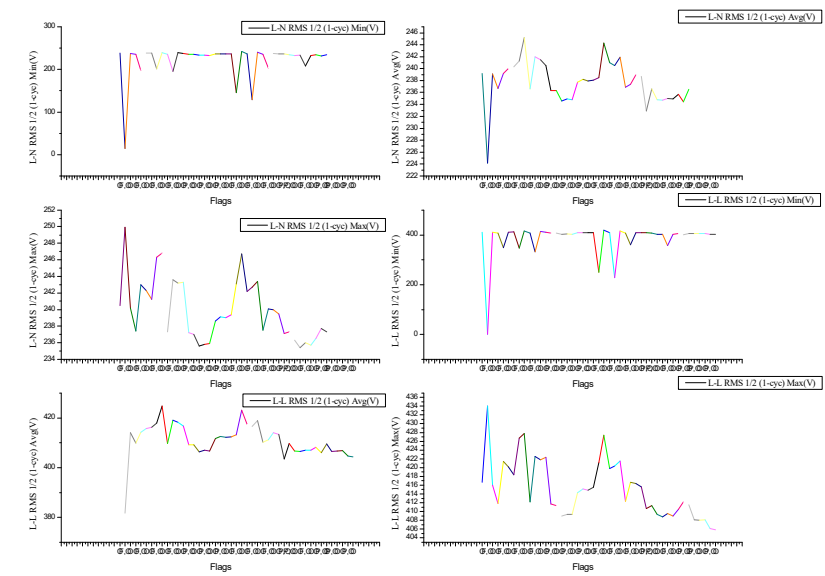

Figure 2. Flag Event vs Drive Input Voltage Behaviour 


\subsection{A Study of Flag Occurrences and Frequency Behaviour:}

Figure 3 shows the frequency analysis of the flag incident. There is not much difference in the frequency values during flag events or pre- and post-flag instants, as seen in the figure. During the F,O instant, the grid system's frequency does not fluctuate considerably, either below or above standard.

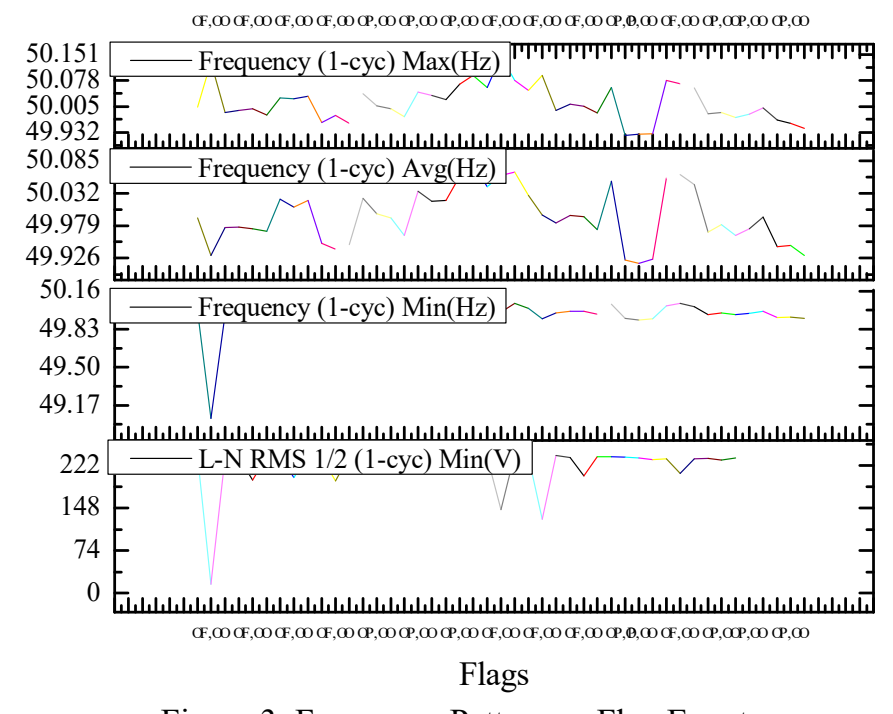

Figure 3. Frequency Pattern vs Flag Event

Figure 4 shows the RMS value of the voltage during the under-frequency flagged event moment and illustrate the RMS voltage waveforms at the beginning and conclusion of the flag event moment. 


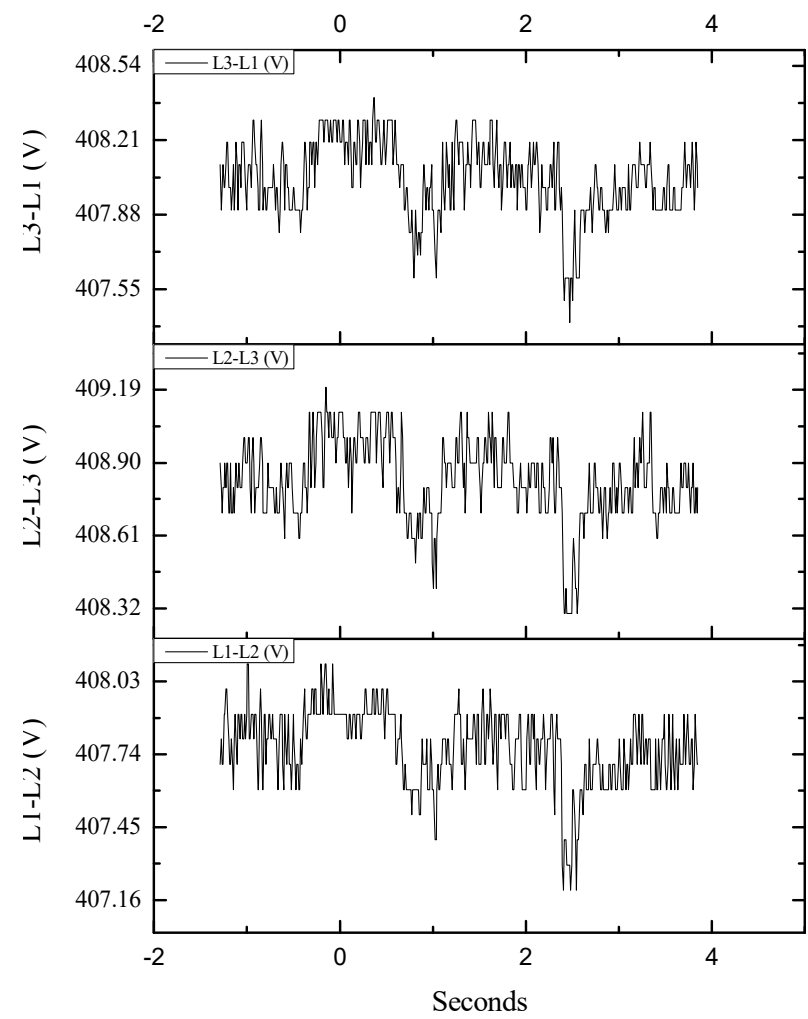

Figure 4. Behavior of RMS voltage during alert occurrence begin and end

Figure 5 also shows the waveform at the conclusion of the under-frequency flag moment. There's no denying that abnormalities in the voltage waveform have a substantial influence on the drive system, as seen in the figure.

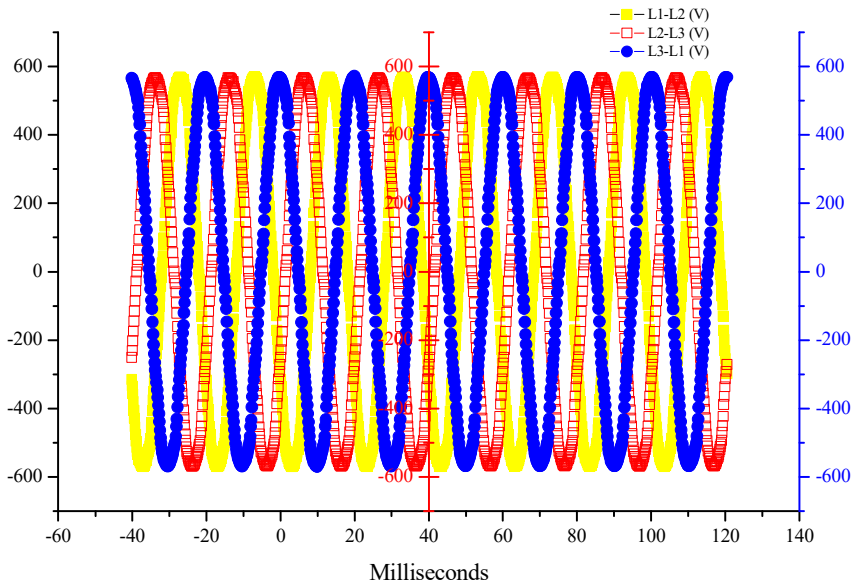

Figure 5. Flag Event Under-Frequency - Voltage waveform (After Restoration of Flag Event) 


\subsection{The Harmonic Distribution of Flag Contingencies}

As mentioned previously, figure 6 depicts the harmonic distortion during the instant of the flag occurrence. The graph demonstrates that harmonics peaked at the H1 level, while the remaining $\mathrm{H} 2, \mathrm{H} 3$,...etc., stay within the limitations. Additionally, identical to the prior underfrequency flag event, this flag event exhibits the same RMS value distortion and post-event voltage waveform, as seen in figure 7 and 8 .

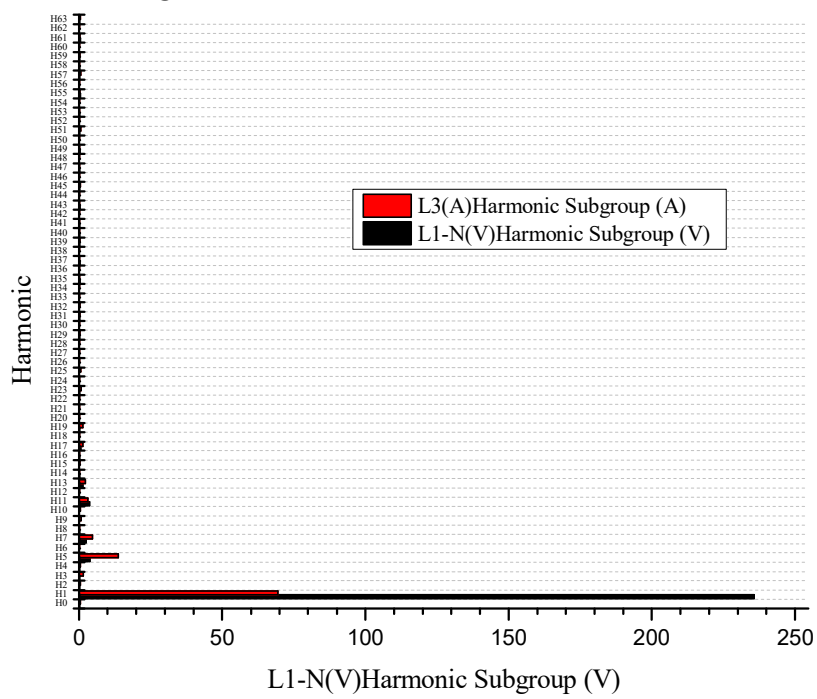

Figure 6. Voltage and Current Distortion during Flag Event

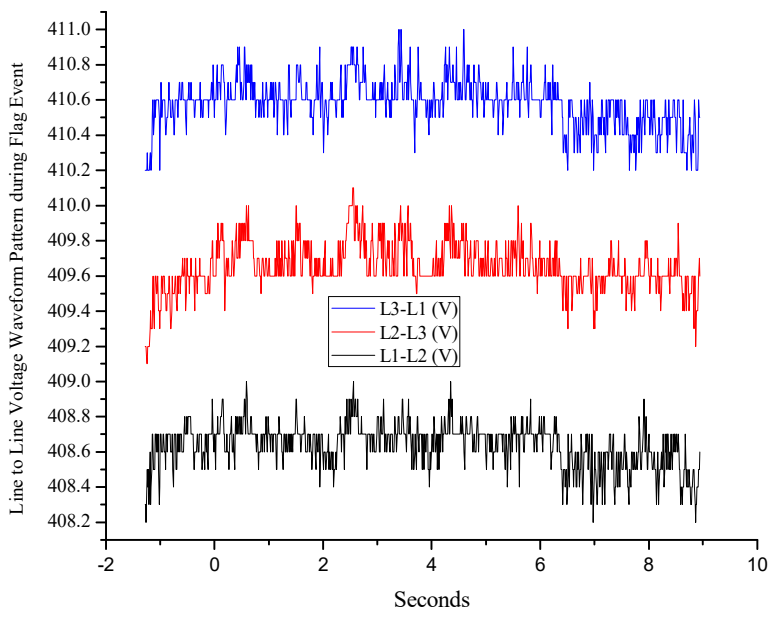

Figure 7: Behavior of RMS voltage during alert occurrence begin and end(Harmonic Flag) 


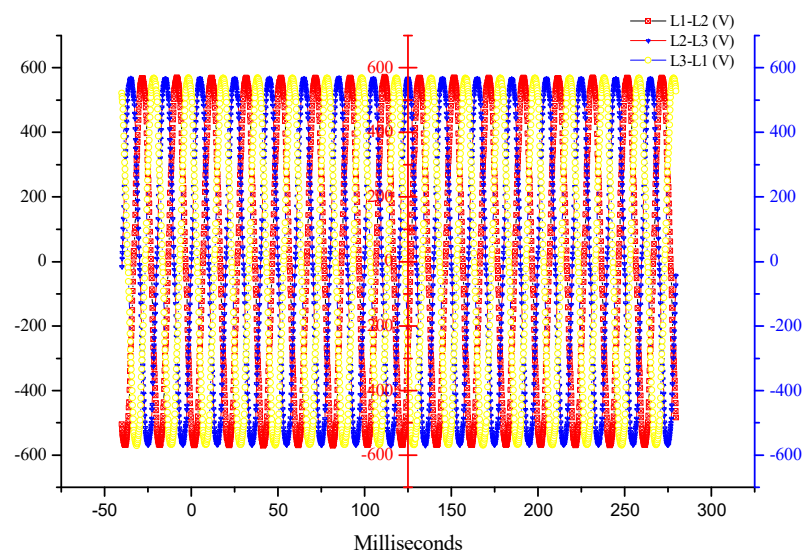

Figure 8. Flag Event Harmonic Flag - Voltage waveform(After Restoration of Flag Event)

In figure 9, the Total harmonic Distortion (voltage and current) is displayed for analysis. Voltage distortion study indicates when the flag event occurs, the harmonic content jumps into the excess range whereas before and following the flag event, the harmonic content stays in the intended range. Additionally, with regards to the current distortion side, it was found that the patterns were identical in figure 9 but they varied in distortion percentage in terms of magnitude.

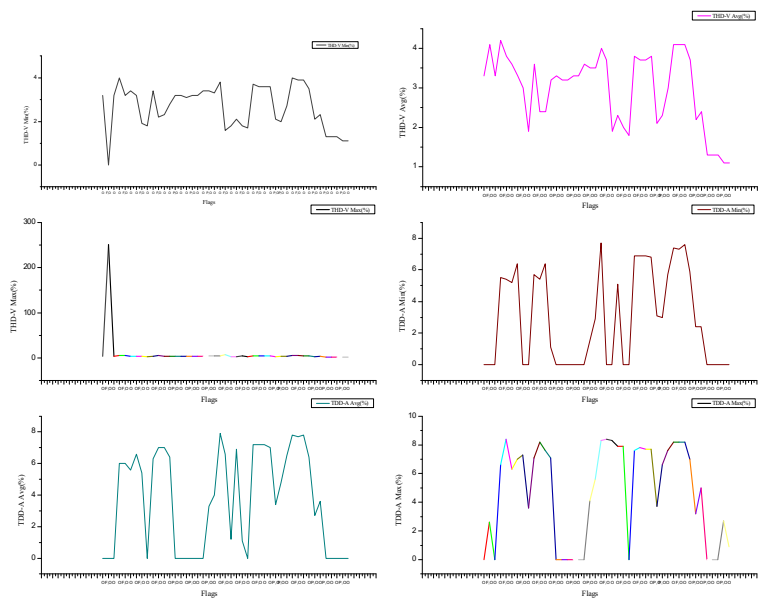

Figure 9. Flag Event vs Percentage Total Harmonic Distortion (Maximum, Average, Minimum)

3.6 Negative Sequence Voltage Trend Relative to Flag Incidence

At typical grid system behaviour, i.e. during the "Flag - O" moment, the negative sequence voltage remains within the grid system's nominal range. It behaves like normal working circumstances when the "Flag - F,O" switch is engaged in figure 10. As a result of the manufacturer's graphical depiction, we were unable to detect any abnormal activity in related to negative sequence value during the flag defect event $\mathrm{F}, \mathrm{O}$. 


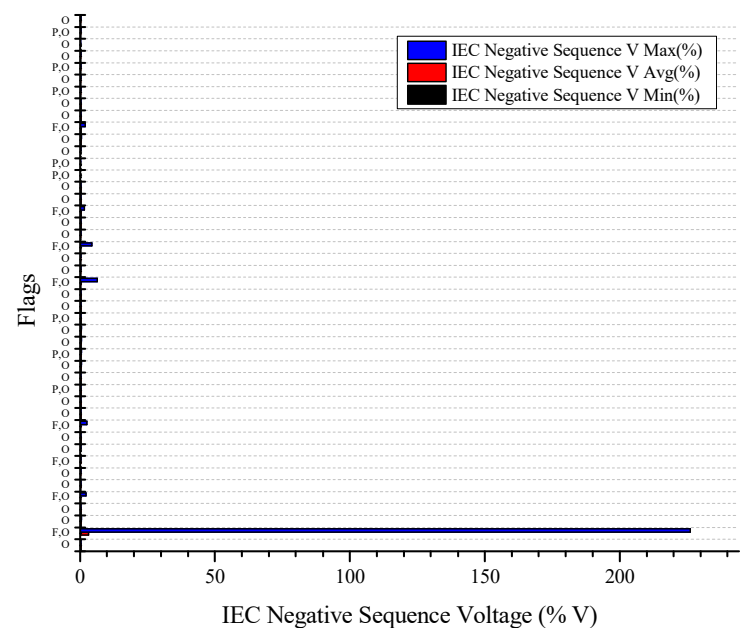

Figure 10. Pattern of IEC Negative Sequence Voltage with Flags Event

\subsection{Zero Sequence Voltage Trend Relative to Flag Incidence}

Figure 11 shows that the zero-sequence voltage factor behaves in a similar way to the negative sequence behaviour described earlier. No aberrant behaviour is documented during $\mathrm{F}, \mathrm{O}$, as well as before and after the $\mathrm{F}, \mathrm{O}$.

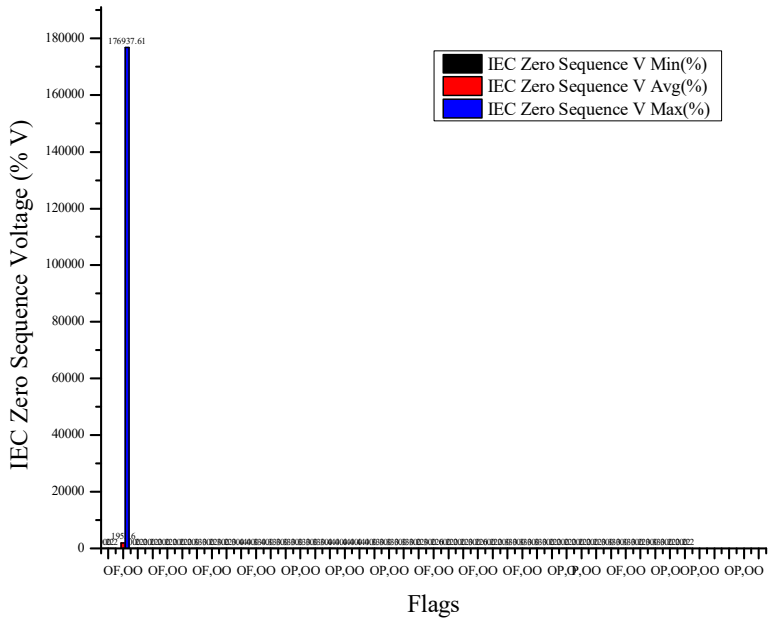

Figure 11. Pattern of IEC Zero Sequence Voltage with Flag Event

3.8 Zero and Negative Sequence Current Trend Relative to Flag Incidence

Figures 12 indicate that, when compared to voltage patterns, the shown current side behaviour displays some dispersed patterns for both zero and negative sequence current values, as can be seen. As can be seen from the graph below, the dispersed effects persist even before and after the flag event F,O. After all, it's difficult to predict with certainty whether or not, when a defect or trigger event happens, this particular parameter will exhibit some distinctive patterns in the drive section. 


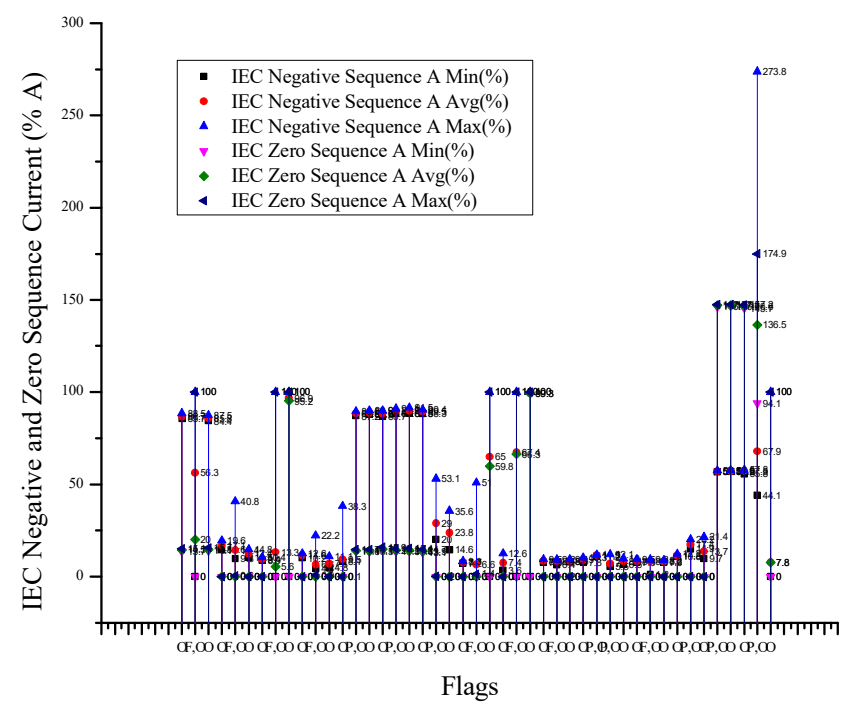

Figure 12. Pattern of IEC Zero, Negative Sequence Current with Flag Event

3.9 Real, Reactive and Apparent Power Trend Relative to Flag Incidence

Fault occurrence instant $(\mathrm{F}, \mathrm{O})$, before and post occurrence "O" do not exhibit any distinctive behaviour for the parameter. Figures 13, 14 and 15 show that actual and reactive power recorded a majority of negative values during 'O', 'F,O', and apparent power recorded a majority of positive values throughout the test phase and drive section. Positive and negative graphs show the respective highest, average, and lowest values for each category. It's clear from this study of these parameters that no special patterns are noticed when a problem occurs in the drive section.

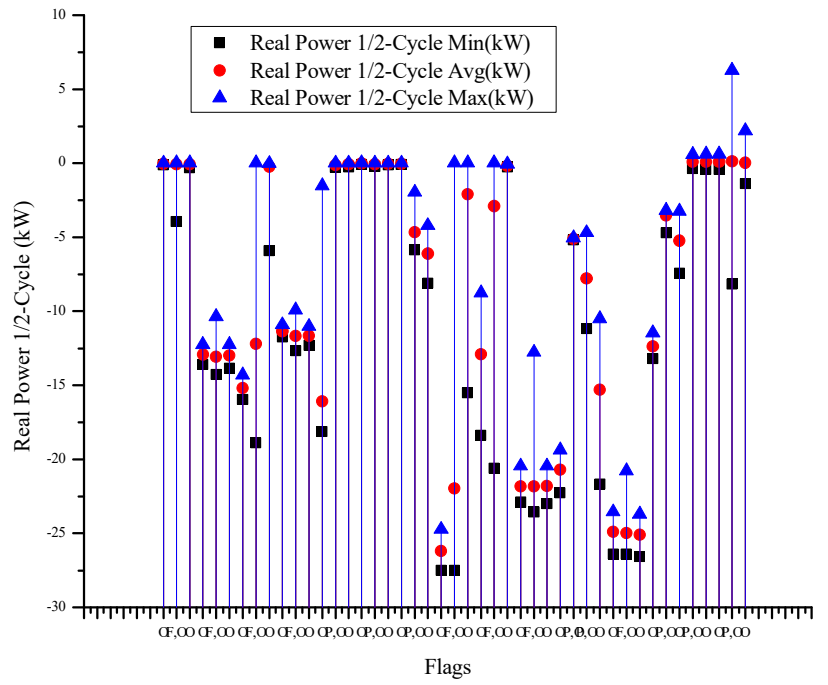

Figure 13. Pattern of Real Power Recorded with Flag Event 


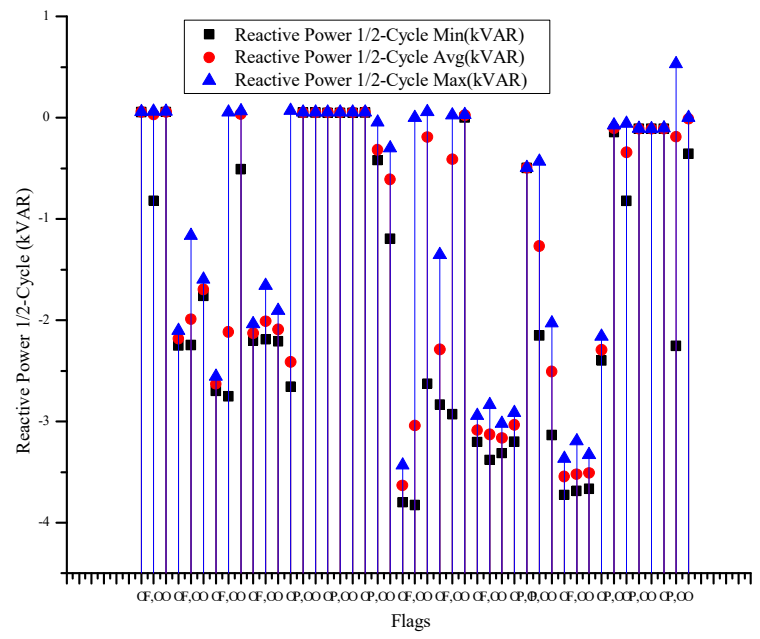

Figure 14. Pattern of Reactive Power Recorded with Flag Event

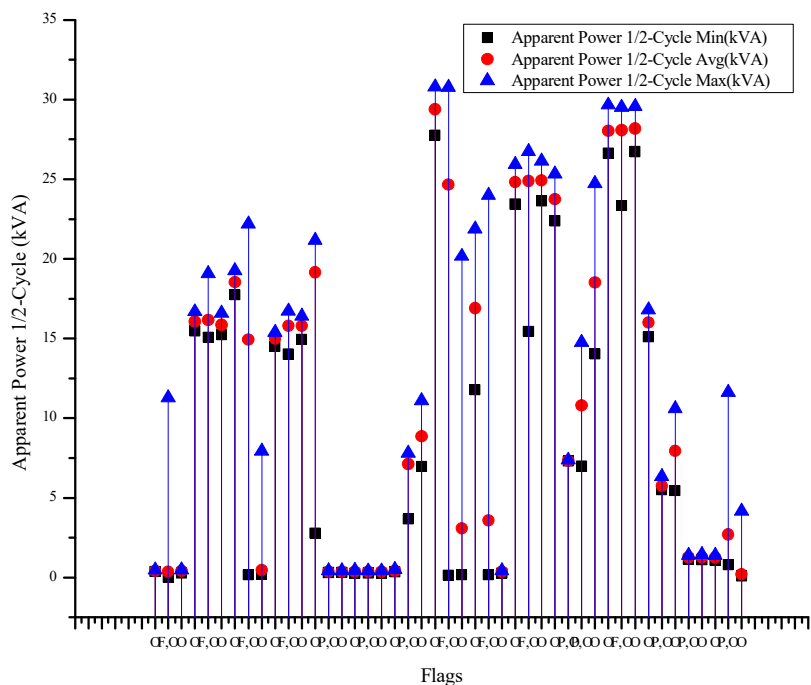

Figure 15. Pattern of Apparent Power Recorded with Flag Event

3.10 Definite Analysis of the Flag Event Difference Versus Parameter Comparison.

These three comparison graphs, figures 16,17 , and 18, demonstrate unequivocally that other indicators do not differ significantly from their typical patterns during the occurrence of the flag. Voltage is the sole measure that exhibits a changing pattern throughout the test phase, although current, frequency, and THD exhibit no abnormal behaviour when their magnitudes are compared before and after 'F,O.' According to this study's findings, grid-side modifications have a detrimental effect on the drive section, which in turn has a detrimental effect on industrial operations. Any modification in parametric behaviour identified within the inverters drive segment is critical for this phase of the study to be successful. Therefore, let us continue with that research, since the upfront rectifier part of the drive section is the first component to be affected by these grid side changes. It was chosen to replicate this rectifier portion using 
MATLAB in order to determine what occurs when an unexpected and anomalous voltage magnitude is recorded at the drive section's input.

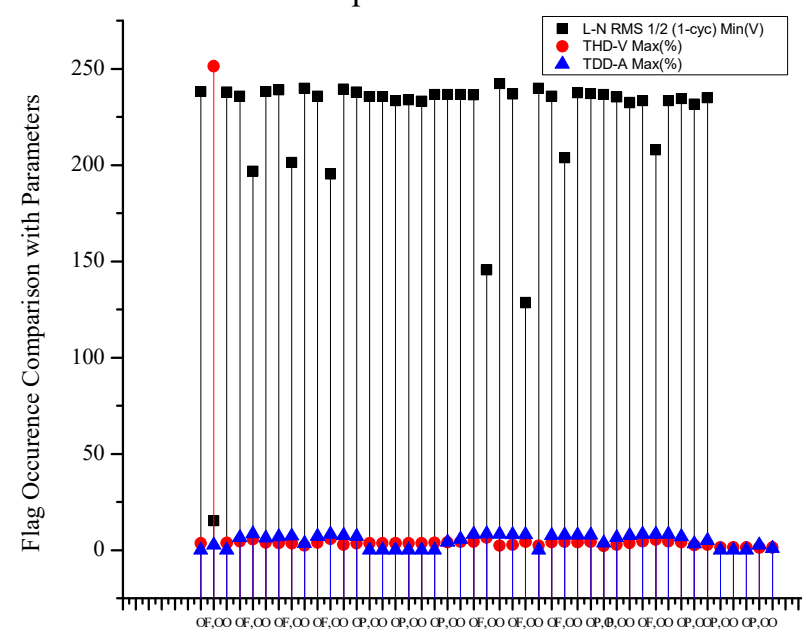

Flags

Figure 16. Analysis of Flag Event Occurence and Harmonic Distortion Maximal Performance

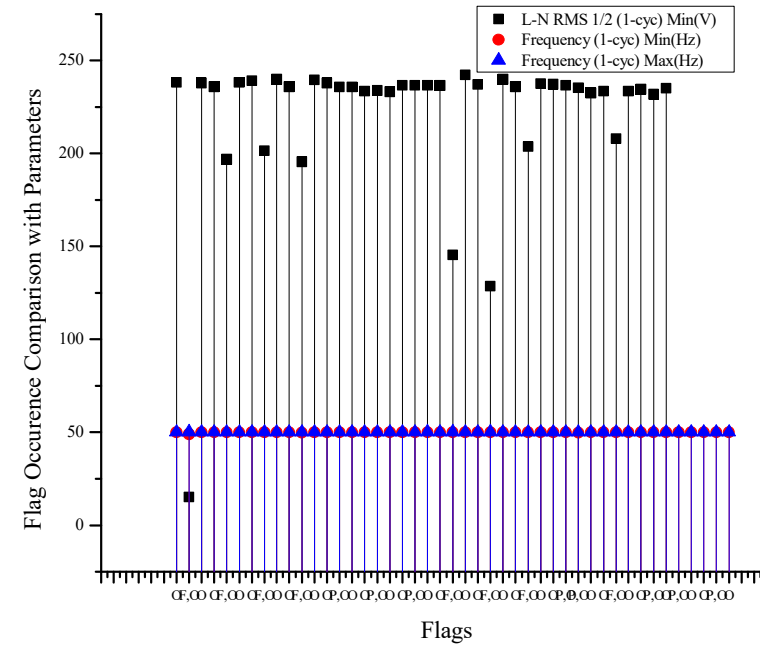

Figure 17. Analysis of Flag Event Occurrence and Frequency Patterns 


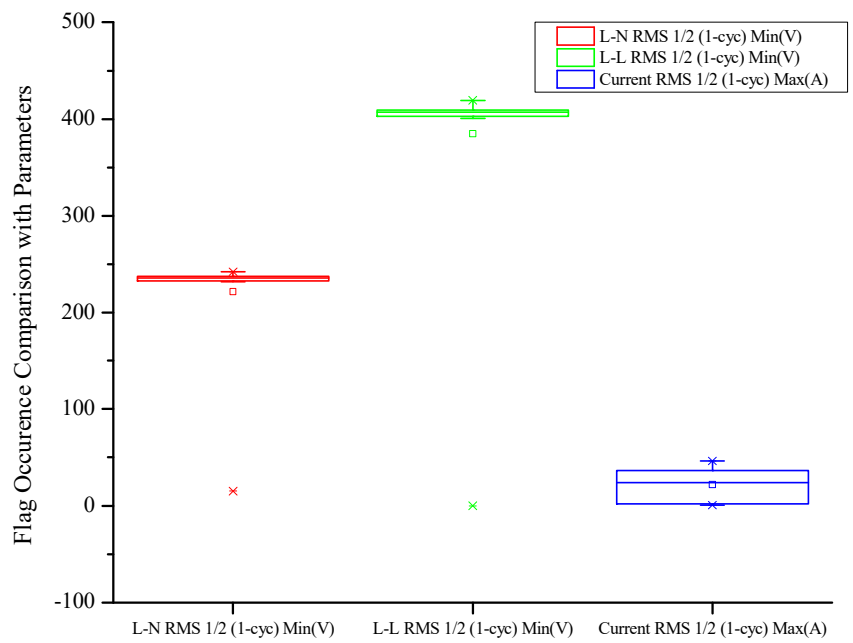

Figure 18. Analysis of Flag Event Occurrence and Three Phase Voltage Performance

\section{Conclusion}

The textile industry was selected for inducting monitoring of various grid network metrics in order to conduct drive failure analysis. Various parametric concerns were analysed with graphical user representation based on data from the test period. Every parameter in the grid network was taken into account in order to find the important or key parameter that failed.That distinct limits do not display any anomaly in their examples during the event "F,O" has solid confirmation.During the 'F,O' event, the voltage parameter is the only one that fluctuates in design; looking at its magnitudes before and after the ' $\mathrm{F}, \mathrm{O}$ ' occasion shows nothing unusual. The prediction procedure is unreliable because it relies on all the above-mentioned events to infer the other grid network parameters and the electrical drive system. Furthermore, just the voltage value anomaly occurs during $\mathrm{F}, \mathrm{O}$, and all other parameters stay normal even at the same instant. In light of the foregoing, implementing a hybrid artificial intelligence method in the drive section will be the best choice for ensuring a dependable and safe operation; this will be described in further detail in the future research work.

\section{References}

[1] D. Dufournet, "IEC and IEEE Standards for High-Voltage Switchgear and Controlgear Present Situation and Future Evolution IEC and IEEE Standards for High-Voltage Switchgear and Controlgear Content," 2008.

[2] I. S. by IEC, "INTERNATIONAL STANDARD INTERNATIONALE ISO Vocabulary," vol. 2001, 2001.

[3] J. Y. Chan and J. V. Milanović, "Severity indices for assessment of equipment sensitivity to voltage sags and short interruptions," 2007 IEEE Power Eng. Soc. Gen. Meet. PES, pp. 1-7, 2007, doi: 10.1109/PES.2007.385457.

[4] P. Wei, Y. Xu, Y. Wu, and C. Li, "Research on classification of voltage sag sources based on recorded events," CIRED - Open Access Proc. J., vol. 2017, no. 1, pp. 846-850, 2017, doi: 10.1049/oap-cired.2017.0907. 
[5] V. Bolgova, A. Leonov, and D. Charkov, "Influence of VFD parameters on voltage stresses in low voltage windings," 2016, doi: 10.1109/RTUCON.2016.7763153.

[6] V. A. Skolota and G. S. Zinovev, "Detecting voltage swell, interruption and sag," Int. Conf. Young Spec. Micro/Nanotechnologies Electron Devices, EDM, vol. 2018-July, no. section 3, pp. 606-611, 2018, doi: 10.1109/EDM.2018.8434940.

[7] D. H. Tourn, J. C. Amatti, J. C. Gómez, and E. F. Florena, "Behavior of the scheme source Capacitor - Induction motor when voltage sags and short interruptions take place," 2006 IEEE PES Transm. Distrib. Conf. Expo. Lat. Am. TDC'06, no. 5800, pp. 1-5, 2006, doi: 10.1109/TDCLA.2006.311576.

[8] M. Demir, M. Iltir, and A. B. Yildiz, "Determination of the effect of short-term interruptions in mains voltage on the reliability of consumer electronics products," Conf. Proc. - 2017 17th IEEE Int. Conf. Environ. Electr. Eng. 2017 1st IEEE Ind. Commer. Power Syst. Eur. EEEIC / I CPS Eur. 2017, 2017, doi: 10.1109/EEEIC.2017.7977551.

[9] M. Al-Badri, P. Pillay, and P. Angers, "A Novel Technique for in Situ Efficiency Estimation of Three-Phase im Operating with Unbalanced Voltages," IEEE Trans. Ind. Appl., vol. 52, no. 4, pp. 2843-2855, 2016, doi: 10.1109/TIA.2016.2533599.

[10] M. Messiha, C. Baraket, A. Massoud, A. Iqbal, and R. Soliman, "Dynamic voltage restorer for voltage sag mitigation in oil \& gas industry,” 2015 1st Work. Smart Grid Renew. Energy, SGRE 2015, 2015, doi: 10.1109/SGRE.2015.7208717.

[11] D. K. Rachev S, Lyubomir Dimitrov, "Study of the Influence of Supply Voltae on the Dynamic Behavior of induction Motor Low Voltage Drive," Int. Sci. J. "Machines, Technol. Mater., vol. 209, no. 5, pp. 206-209, 2017.

[12] J. Meyer, A. M. Blanco, M. Domagk, and P. Schegner, "Assessment of Prevailing Harmonic Current Emission in Public Low-Voltage Networks,” IEEE Trans. Power Deliv., vol. 32, no. 2, pp. 962-970, 2017, doi: 10.1109/TPWRD.2016.2558187.

[13] F. L. Hoadley, R. F. McElveen, and T. R. Obermann, "Application considerations for operating VSI-FED MV motors in hazardous locations,” IEEE Trans. Ind. Appl., vol. 53, no. 2, pp. 16561668, 2017, doi: 10.1109/TIA.2016.2630019.

[14] Prabha, S. Lavanya, M. Surendar, and M. Neelamegam. "Experimental investigation of ecofriendly mortar using industrial wastes." Journal of Green Engineering 9.4 (2019): 626-637.

[15] M. Tholkapiyan, A.Mohan, Vijayan.D.S , "A survey of recent studieson chlorophyll variation in Indian coastal waters", IOP Conf. Series: Materials Science and Engineering 993 (2020) 012041 , doi:10.1088/1757-899X/993/1/012041. 